\title{
Dos interesantes contaminantes fúngicos filamentosos en muestras clínicas positivas a Trychophyton rubrum. Una situación de curiosidad morfológica
}

\author{
Two interesting filamentous fungal contaminants in clinical samples positive to \\ Trychophyton rubrum. A situation of morphological curiosity
}

\author{
Dr. Eduardo Piontelli Laforet ${ }^{1^{*}}$ \\ TM. Peggy Vieille Oyarzo ${ }^{2}$ \\ TM. Yerko Leiva Salazar ${ }^{2}$ \\ ${ }^{1}$ Profesor Emérito de Micología, Universidad de Valparaíso. \\ ${ }^{2}$ Laboratorio Diagnóstico Microbiológico UVCLIN, \\ Escuela de Medicina, Universidad de Valparaíso. \\ *Autor para correspondencia: eduardopiontelli@ hotmail.com
}

RECIBIDO: 24 de mayo 2021

APROBADO: 2 de julio 2021

DOI: 10.22370/bolmicol.2021.36.1.2907

Palabras claves: Dermatofitosis, contaminantes, Metarhizium, Monascus.

Keywords: Dermatophytoses, contaminants, Metarhizium, Monascus.

\section{RESUMEN}

Las dermatofitosis corresponden a un grupo de enfermedades micóticas comunes en piel y fanéreas, donde Trichophyton rubrum es el agente causante más frecuente a nivel mundial y presente en nuestros 2 casos de pacientes masculinos con estas micosis, una en uñas y la otra en piel. Sin embargo, el enfoque de esta publicación se basa principalmente en la presencia de 2 interesantes contaminantes (uno en cada caso clínico) presentes solo en los cultivos de las primeras siembras como saprófitos y por ende como propágulos de dispersión, asociados al ambiente y sin intervención clínica demostrada en ambas micosis. La descripción morfofisiológica de estos 2 contaminantes Metarhizium purpureogenum(similis) y Monascus ruber fue más bien una curiosidad esencial que el micólogo clínico adquiere en su contínua formación y ante la posibilidad de infecciones mixtas, pudiendo conjugar sus hallazgos junto al análisis taxonómico y los factores geográficos y edáficos asociados a su distribución.

\begin{abstract}
Dermatophy toses belongs to a group of common mycotic diseases in skin and pharynals, where Trichophyton rubrum is the most frequent causative agent worldwide and present in our 2 cases of male patients with these mycoses, one in nails and the other in skin. However, the focus
\end{abstract}


Dos interesantes contaminantes fúngicos - Piontelli E. et al.

of this publication is mainly about the presence of 2 interesting contaminants (one in each clinical case) present only in the crops of the first sowings as saprophytes and therefore as dispersal propagules, associated with the environment and without clinical intervention demonstrated in both mycoses. The morphophysiological description of these 2 contaminants, Metarhizium purpureogenum (similis) and Monascus ruber was rather an essential curiosity that the clinical mycologist acquires in his continuous training and in the face of the possibility of mixed infections, being able to combine his findings together with the taxonomic analysis and the geographic and edaphic factors associated with its distribution.

\section{INTRODUCCIÓN}

La identificación de hongos patógenos u oportunistas o contaminantes ya sea en lesiones superficiales o profundas todavía se basa en los laboratorios clínicos, principalmente mediante aportes morfofisiológicos, sin embargo, en los 2 últimos decenios se han podido complementar cuando es necesario, mediante espectrometría de masas o secuenciación de ADN (Alexander et al. 2020). Comúnmente, en los aislamientos fúngicos desde cultivos de muestras humanas superficiales de piel y fanéreas positivas a Trichophyton rubrum, puede apreciarse la presencia de hongos no pertenecientes a los dermatofitos, una situación que puede considerarse como: 1) Meros contaminantes saprófitos que no intervienen en la micosis (como en nuestro caso), debido a su presencia como propágulos de dispersión, asociados al ambiente frecuentado en las actividades comunes del paciente afectado, y 2) Como integrante de una posible onicomicosis mixta, considerando que, la infección primaria del dermatofito puede generar el microambiente necesario para que el hongo no dermatofito tengan la oportunidad de estar presente como oportunista secundario o incluso desplazarlo si fuese capaz de crecer a $37^{\circ} \mathrm{C}$ (López Olga y M Torres-Rodríguez,1999; Alexander GómezSáenz y Roberto Arenas 2020). Esta última situación no se considera en nuestra investigación.

El diagnóstico de lesiones duales es complejo, mientras $\boldsymbol{T}$. rubrum es un patógeno antropofílico, el oportunista acompañante es generalmente un típico integrante de la microbiota ambiental como lo son las especies de Aspergillus, Fusarium, Scopulariopsis, Acremonium (entre muchos otros) (López Olga y M Torres-Rodríguez,1999). Más de 30 especies no integrantes de los dermatofitos se han implicado como causantes de dermato $\mathrm{u}$ onicomicosis (Alexander et al. 2020). El preciso papel de estos hongos es muchas veces controvesial en la manera de interpretar estos hallazgos clínicos (Gupta et al. 2012). Esta temática en nuestros casos está más allá del alcance de este artículo debido a que solo nos referiremos a 2 contaminantes integrantes de los Ascomycetes identificados solo por morfofisiológía como integrantes del género Metarhizium (Paciente 1) y Monascus (Paciente 2). Teniendo en cuenta, que estos microorganismos pueden estar presentes en áreas corporales superficiales como microbiota derivada de ambientes diversos y sin una implicancia clínica, su presencia puede ser solo de interés para el micólogo desde varios puntos de vista, ya sea como: un aporte en su curiosidad taxonómica, conocer su dispersión geográfica o un interés de búsqueda bibliográfica que permita relacionarlos o no asociados a potenciales posibles casos de oportunismo (Gupta et al. 2012).

\section{METODOLOGÍA}

Se obtuvieron muestras clínicas de 2 pacientes de sexo masculino mediante raspado de lesiones 
Dos interesantes contaminantes fúngicos - Piontelli E. et al.

en piel y uñas: El primer caso (Paciente 1) correspondió a un hombre de 42 años con onicolisis en uñas de los pies. Con un bisturí estéril se obtuvo descamación del borde distal y lateral subungueal y de las zonas más proximales de la lámina ungueal del primer dedo del pie derecho ("Hallux"). El segundo caso (Paciente 2), fue en un hombre de 55 años que presentaba descamación interdigital de los dedos de la mano derecha entre el índice y el dedo medio. Los raspados de ambos pacientes se mantuvieron en placas estériles de Petri pequeñas hasta su procesamiento. Para el examen microscópico de las escamas obtenidas, éstas se colocaron entre porta y cubreobjeto, agregando hidróxido de potasio $(\mathrm{KOH})$ al $20 \%$, para observar luego al microscopio la presencia de micelios o levaduras en un lapso de media hora. En ambos casos clínicos se observó la presencia de trozos de micelios septados, ramificados formando artroconidios. Los cultivos de ambos casos clínicos se incubaron a temperaturas de $25^{\circ} \mathrm{C}$ y $37^{\circ} \mathrm{C}$ mediante siembras en 2 tubos de agar Sabouraud $10 \%$ (2g. glucosa, $1 \mathrm{~g}$. peptona) y 2 tubos de agar Lactimel, todos ellos con cloranfenicol $200 \mathrm{mg} / \mathrm{L}$ y con tiempos de incubación de 14 días a las 2 temperaturas mencionadas.

\section{Interpretación de los cultivos.}

De los cultivos micológico a ambas temperaturas se obtuvieron varios aislamientos que permitieron llegar al diagnóstico clínico definitivo de tinea pedis (paciente 1) y tinea manum (paciente 2), causadas ambas por $\boldsymbol{T}$. rubrum por la presencia de colonias planas a ligeramente elevadas, de color blanco a crema, vellosas, con un reverso de color marrón amarillento a rojo vino y una morfología clásica macro y microscópica correspondiente a este taxón. Se destaca la presencia en Sabouraud 10\% y solo en los tubos a $25^{\circ} \mathrm{C}$ de un contaminante en la parte superior del agar inclinado en los cultivos de ambos pacientes con una colonia reducida de color ocráceo en el paciente 1 y un color cafesoso en el paciente 2, cuyas características microscópicas no se pudieron asociar claramente a algún género común. En los tubos a $37^{\circ} \mathrm{C}$, solo se presentaron colonias de $T$. rubrum. Aceptando nuestra curiosidad morfológica frente a estos contaminantes y poder confirmar una posible nueva presencia en las muestras, se volvieron a resembrar los rapados dérmicos de ambos pacientes en los medios especificados anteriormente, a ambas temperaturas, obteniéndose nuevamente solo positividad para $\boldsymbol{T}$. rubrum y la ausencia en ambos casos de los contaminantes.

Nuevos aislamientos. Al no reconocer el género de estos 2 "allegados", se sembraron ambos en PDA por $7-10$ días a $25^{\circ} \mathrm{C}$ y se efectuaron preparaciones con lactofenol y etanol al $70 \%$ para su observación microscópica, la cual presentó en el paciente 1, abundantes esporodoquios con producción de cadenas de conidios blásticos en largas columnas de color ocráceo y un pigmento difusible rojo púrpura en el medio que empezó a manifestarse a los 3-4 días. Estos datos morfológicos permitieron su ubicación como un integrante del género Metarhizium (no hubo desarrollo a $37^{\circ} \mathrm{C}$ ). En PDA pudo observarse también contaminación exógena dispersa de cepas del género Cladosporium (Fig.1). En el paciente 2 se apreció la presencia de cleistotecios cafesosos abundantes, ascos y ascosporas y un anamorfo blástico asociado que producía cadenas cortas de conidios de base trunca permitiendo reconocer un integrante del género Monascus que crecía muy bien también a $37^{\circ} \mathrm{C}$ (con un máximo de $\left.45^{\circ} \mathrm{C}\right)$.

Reconociendo lo poco común de estos 2 géneros en muestras clínicas, efectuamos nuevas resiembras de ambas cepas en 3 puntos sobre placas de $90 \mathrm{~mm}$ en CYA, MEA y YES y en 
Dos interesantes contaminantes fúngicos - Piontelli E. et al.

placas de $50 \mathrm{~mm}$ en CREA a 25 y $37^{\circ} \mathrm{C}$, durante 7 a 14 días para aportar un conjunto de datos morfofisiológicos que permitieran acercarnos a un diagnóstico a nivel de especies. Todos los medios se prepararon de acuerdo a Samson et al. (2010). Los diámetros de las colonias se midieron después de 7 días de incubación y sus características se registraron (presencia de pigmentos solubles, exudados, colores del anverso y reverso de las colonias y el color de micelio). Observaciones microscópicas de la etapa asexual se hicieron a partir de colonias cultivadas en MEA. Se utilizó ácido láctico (60\%) como líquido de montaje y etanol al $70 \%$ para eliminar el exceso de burbujas. El tamaño, la forma y pigmentación de conidios, conidióforos, ascomas, ascos y ascosporas fueron fotografiados en un microscopio óptico Zeiss Axioscope equipado con cámara Canon Powershot G5 DIGIC.

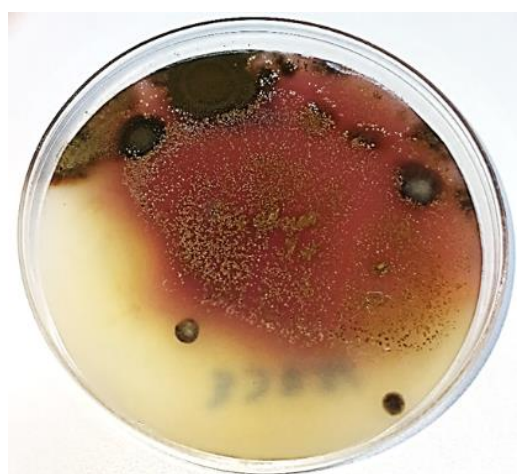

Fig. 1. Siembra en PDA. Colonia granular dispersa, color amarillo ocre (Metarhizium sp.) con fuerte pigmento difusible rojo púrpura asociada al crecimiento conjunto de colonias contaminantes del género Cladosporium.

\section{RESULTADOS}

\section{Diagnóstico Clínico}

El diagnóstico clínico de los 2 pacientes analizados fue de dermatofitosis por $\boldsymbol{T}$. rubrum en uña de los pies (paciente 1) e interdigital en la mano derecha (paciente 2) confirmado por el análisis morfológico de laboratorio de los cultivos a $25^{\circ} \mathrm{C}$ y $37^{\circ} \mathrm{C}$ y las preparaciones microscópicas correspondientes.

\section{Morfología de la colonia Metarhizium}

Colonias en CYA de crecimiento relativamente rápido, $33-34 \mathrm{~mm}$ a 7 días a $25^{\circ} \mathrm{C}$, primeramente, con micelio hialino, luego crema en los bordes, oscureciéndose las colonias hacia la parte central por la formación de conidios. Con centro de la colonia solevantado dando el aspecto de un botón. Desde el 3-4 día se aprecia la producción de pigmento rojo púrpura difusible alrededor de las colonias. Reverso rojo púrpura intenso (Fig.2A). En MEA a $25^{\circ} \mathrm{C}$ colonias de crecimiento levemente mayor que en CYA 34$35 \mathrm{~mm}$, primeramente, hialinas que se tornan rápidamente en tonos canela claro y hacia el centro se aprecia una formación de anillos concéntricos compactos de color canela a ocre debido a la abundante formación de conidios en masas compactas, que tienden a separarse dando un aspecto costroso en regulares cadenas $\mathrm{y}$ apretadas columnas (Fig.3 foto de Lupa a 25 X). La colonia en este medio produce la mayor cantidad de pigmento difusible rojo púrpura a su alrededor y reverso del mismo color (Fig.2B). Crecimiento en YES similar al que se presenta CYA ya sea en las medidas como en la descripción de las colonias, incluso en sus diámetros a los 7 días con la misma formación de pigmento difusible en el anverso y reverso destacando solo un menor diámetro de difusión de éste (Fig. 2C). CREA sin viraje ácido y crecimiento casi imperceptible (Fig. 2D en recuadro). 
Dos interesantes contaminantes fúngicos - Piontelli E. et al.
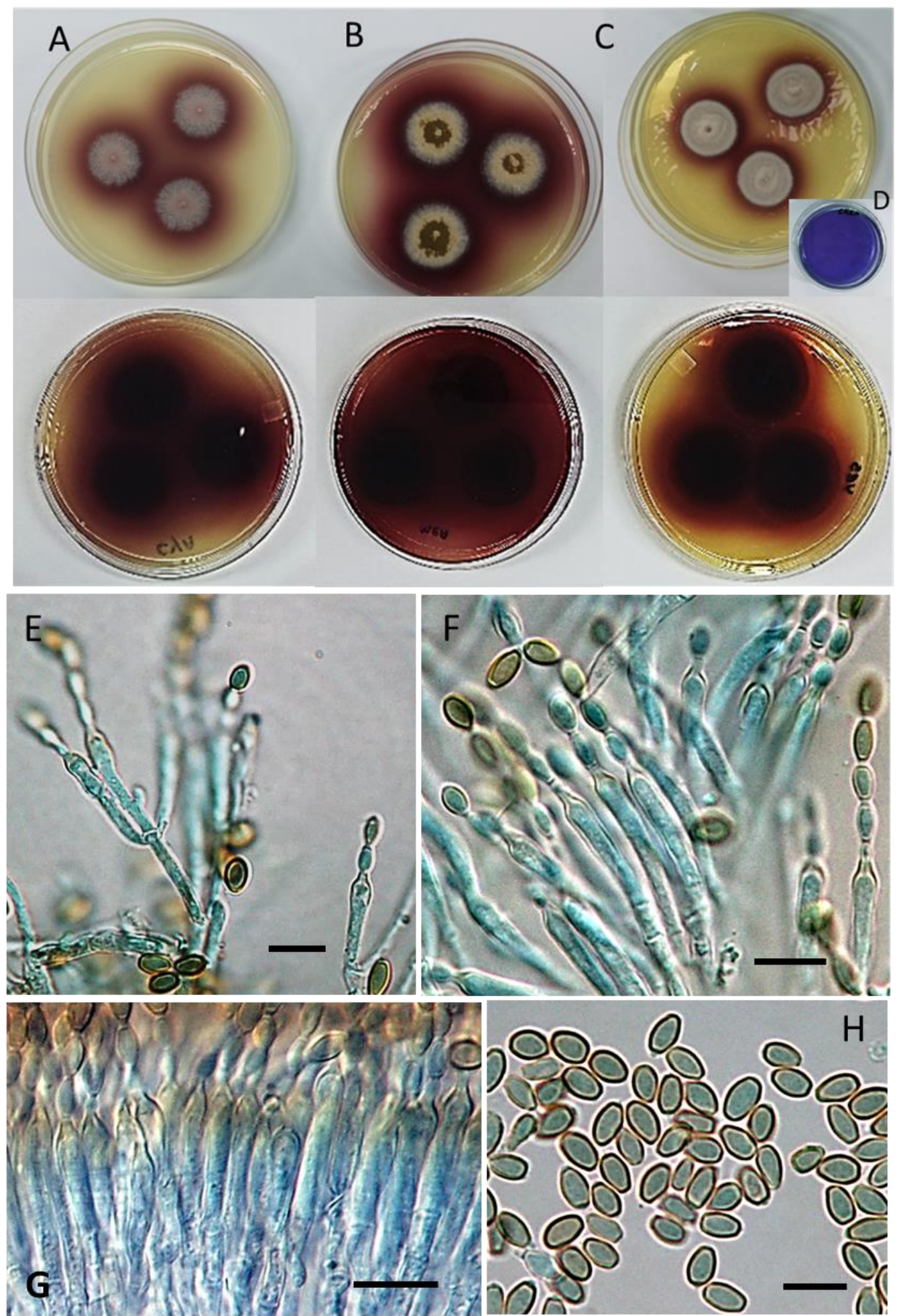

Fig 2. Anverso de las colonias de Metarhizium purpureogenum (similis) a $25^{\circ} \mathrm{C}$ en 7 días. A. CYA, B. MEA, C. YES. Reverso con pigmento difusible rojo púrpura de las colonias a los 9 días en los mismos medios. D. CREA. E-F. Fiálides de cuello angosto y formación de conidios en cadenas basípetas. G. Fiálides y conidios compactados en regulares cadenas y apretadas columnas. H. Conidios lisos, elípticos de base trunca. Barras $10 \mu \mathrm{m}$. 
Dos interesantes contaminantes fúngicos - Piontelli E. et al.

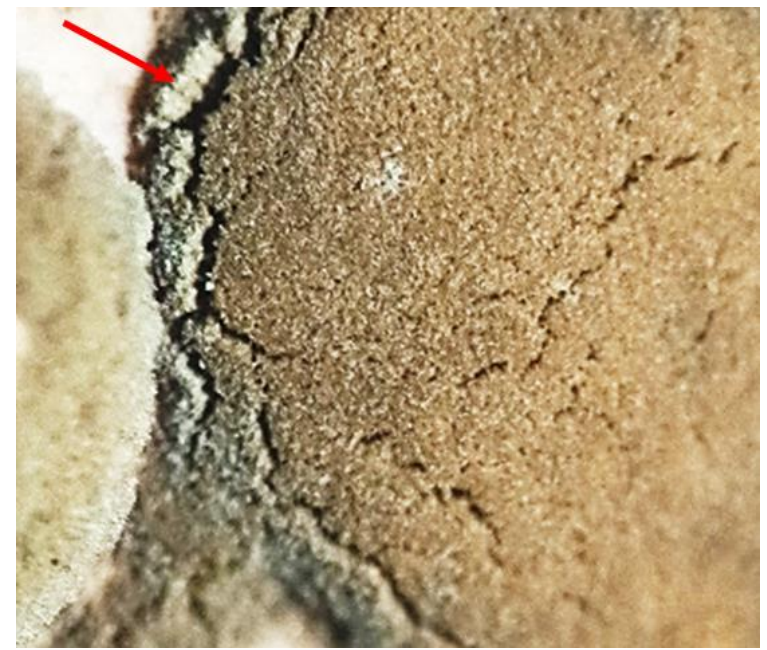

Figura 3. Metarhizium purpureogenum (similis) en MEA a los 7 días. Ampliación bajo lupa estereoscópica de la parte central de la colonia (25X). Nótese (flecha) las columnas de conidios adosadas estrechamente originadas por los esporodoquios basales y las hendiduras costrosas de la comprimida masa conidial.

\section{Morfología de las estructuras reproductivas de Metarhizium.}

Todas las estructuras analizadas fueron en base a las medidas de 20 unidades. Los conidios en MEA 25 a los 7 días son ovoides a elipsoides, lisos, 5,5- $7.0(-8)$ x 3-4 x 4,5 (-5) $\mu \mathrm{m}$ (Promedio $6,1, \mu \mathrm{m}$ (DS 0,6-0,4). Conidios en CYA ovoides a elipsoides lisos 5-8 x 2,5-3,5 $\mu \mathrm{m}$ (media 5,8 x $3,2 \mu \mathrm{m}$ (DS 0,7-0,4). YES semejantes en color y tamaño al CYA. Las fiálides en MEA 25 son cilíndricas que se angostan apicalmente formando cortos cuellos $(\geq 2 \mu \mathrm{m})$ y estrechos $(\leq 2$ $\mu \mathrm{m})$ (Fig-2 F-G), 12-20 x 2,5-3.0 $\mu \mathrm{m}$ (Promedio 16,3, DS 2,1-0,3). En base a las estructuras analizadas y las características morfofisiológicas de los cultivos y el tamaño, forma $y$ pigmentación de los conidios y células conidiógenas se determinó la especie como Metarhizium purpureogenum (similis).

\section{Morfología de las colonias de Monascus.}

Crecimiento en CYA $26 \mathrm{~mm}$ diám. en 7 días a $25^{\circ} \mathrm{C}$, con colonias levemente algodonosas de un color canela claro en los bordes, oscureciéndose levemente hacia el centro. Reverso en CYA semejante al anverso. En MEA, colonias de 35 $\mathrm{mm}$ diám., algodonosas con bordes anchos de un color canela a salmón claro, con el centro café debido a las estructuras de esporulación y con la presencia de surcos en forma radiada. Reverso color salmón. YES, colonias de $22 \mathrm{~mm}$ con bordes filamentosos con grados de naranja oscureciéndose levemente hacia el centro. Centro con tonos rosados de aspecto algodonoso, Reverso igual que el anverso. CREA con crecimiento micelial escaso, poco visible (alrededor de $10 \mathrm{~mm}$ ) sin ácido. MEA $37^{\circ} \mathrm{C}$, colonias de hasta $60 \mathrm{~mm}$ de color café de aspecto granuloso, de bordes angostos blanquecinos que se oscurecen de color café casi desde el borde hacia el centro y presentando visibles surcos radiales desde el centro. Reverso similar en tonos más claros. (Vea Fig. 4 a-b).

\section{Estructuras reproductivas.}

En MEA a $25^{\circ} \mathrm{C}$ a los 10 días. Ascomas cleistoteciales no ostiolados de $28-65 \mu \mathrm{m}$ de diámetro, (promedio 43,2 $\mu \mathrm{m}$ DS.18,3) que nacen únicos en el ápice de una hifa semejante a un pedúnculo que brota en una vesícula, la cual se rodea por ramas de hifas que se fusionan semejando una membrana con un peridio formado por hifas unidas que se tornan café en el tiempo. Grosor de las paredes 2-3,5 $\mu \mathrm{m}$. Ascos prototunicados, evanescentes, subesféricos, octoesporados, ascosporas elipsoidales 5-6,5(-7) x 3,5-4,5 $\mu \mathrm{m}$ (promedio 5,7 $\mu \mathrm{m}$, DS 3,8) de paredes lisas. Conidios obpiriformes a esféricos, de base trunca $8-13 \mu \mathrm{m}$ diám. (promedio $10 \mu \mathrm{m}$, DS 10 x 8,8) que se forman únicos o en cortas cadenas basípetas, de paredes gruesas hialinos a cafesosos (basipetospora-morph). Clamidosporas intercalares raras.

A pesar que la apariencia externa de las colonias es una característica fenotípica esencial, la morfología de $M$. ruber es extremadamente variable en el color y tamaño de las colonias En 
Dos interesantes contaminantes fúngicos - Piontelli E. et al.

base a los datos de cultivo y la morfofisiología del anamorfo y teleomorfo, junto a la literatura analizada, se clasifica a la especie como Monascus ruber Tiegh.

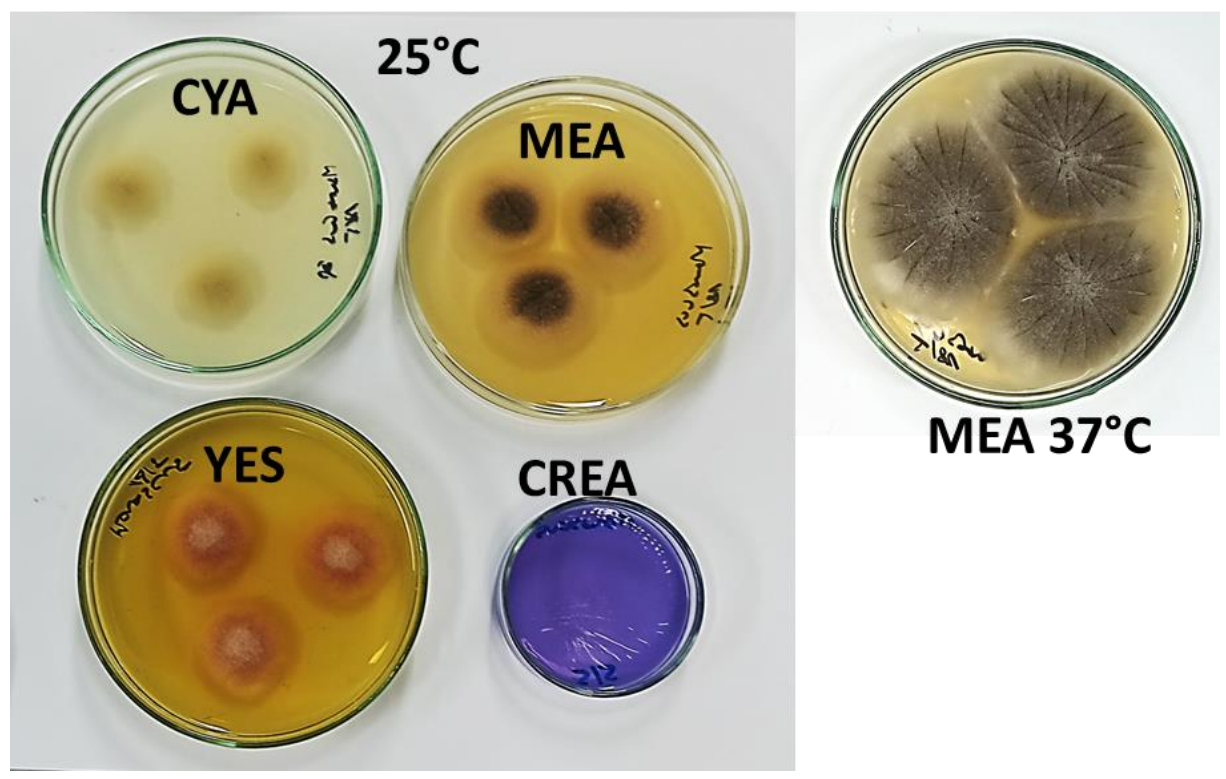

FIG 4A. Colonias de Monascus ruber Anverso. Colonias en CYA, MEA, YES y CREA con crecimiento escaso y poco visible indicado con la flecha, sin ácido. $\mathbf{M E A ~} 37^{\circ} \mathbf{C}$, colonias surcadas.

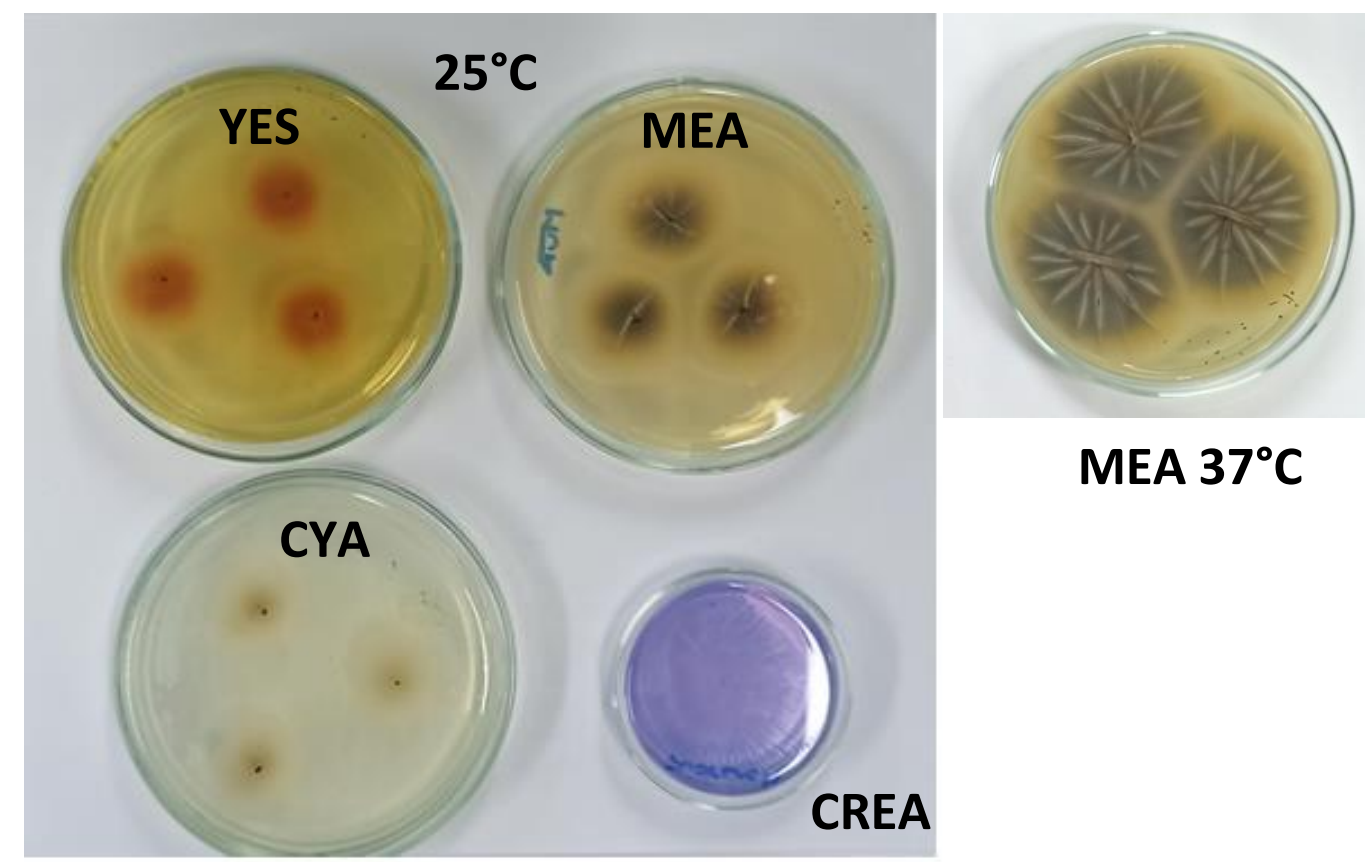

Fig 4B. Colonias de Monascus ruber. Reverso. CYA semejante al anverso. YES Naranja más claro en los bordes, MEA. Bordes crema con centro café. CYA. Crecimiento poco visible sin ácido. MEA $37^{\circ} \mathbf{C}$ bordes crema, centro café con surcos fuertemente radiados color crema. 
Dos interesantes contaminantes fúngicos - Piontelli E. et al.
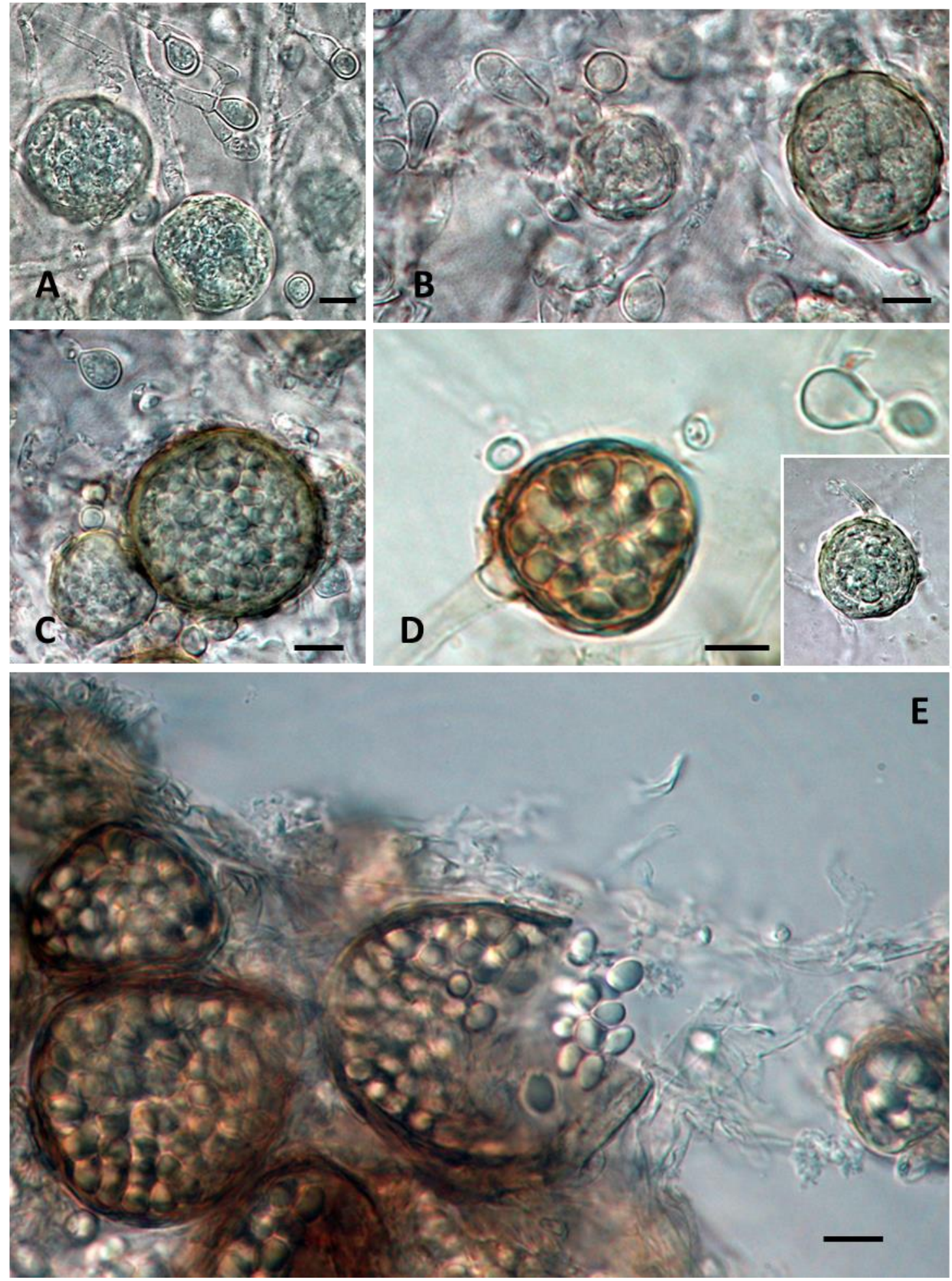

Fig. 4. Monascus ruber. A-B.C. Cleistotecios jóvenes con delimitación de ascos y algunos conidios D. Cleistotecio maduro con ascosporas, pedúnculo de soporte semejante a hifa (también en recuadro) y un conidio. E. Cleistotecios y ascosporas libres. Barras $10 \mu \mathrm{m}$. 
Dos interesantes contaminantes fúngicos - Piontelli E. et al.

\section{DISCUSIÓN}

El género Metarhizium Dikarya, Ascomycota Pezizomycotina, Sordariomycetes Hypocreomycetida, Hypocreales, Clavicipitaceae (Raymond R y Pihet EM. 2008), incluye especies saprófitas del suelo comúnmente aisladas de la rizósfera, suelos cultivados o naturales en regiones tropicales como temperadas (Xinggang Liao et al, 2014; Donald W.Roberts \& Raymond J.St. Leger 2007). Las especies de Metarhizium son ampliamente conocidas por su potencial entomopatogénico letal en artrópodos y por ser uno de los géneros con diversos ciclos de vida y estados reproductivos morfológicos asexuales (Luangsa-ard, S. et al.2017). Antes del año 2000, la clasificación de Metarhizium se basó principalmente en caracteres morfológicos, situación que fue solucionándose mediante el empleo de la biología molecular, la cual permitió reconocer las especies crípticas y la diversidad genética en este género (F. Driver, et al. 2000). En un esfuerzo basado en el concepto de un solo nombre para los hongos Kepler et al. (2014) proponen la supresión de Metacordyceps en favor de Metarhizium pero retienen Pochonia y crean el nuevo género Metapochonia para acomodar los miembros de Pochonia que no se agrupan con la especie tipo. Las especies de Chamaeleomyces y Nomuraea (excluyendo $N$. atypicola y Paecilomyces viridis) fueron transferidas también a Metarhizium, reconociendo un total de 34 especies. En los tiempos actuales con el aumento de datos secuenciales, se reconocen 69 especies del género aumentando la asociación de hospedadores, la ecología y distribución de nuevas especies desde: Argentina, Brasil, China, Japón y Tailandia (S. Mongkolsamrit. et al. 2020).

Los agentes entomopatógenos más estudiados entre los hongos corresponden principalmente a los géneros Beauveria, Metarhizium y Paecilomyces (De A, et al. 2014). Según Nishi et al. (2017) M. purpureogenum representa un linaje único en el género, con una afinidad débil por M. novazealandicum. Todos los aislamientos disponibles de esta especie se recuperaron del suelo y se desconocen las asociaciones de hospedadores de esta nueva especie. Sin embargo, la recuperación exitosa de $M$. purpureogenum en muestras de suelo con termitas, así como la infección experimental de larvas de gusanos de seda demuestran su potencial como entomopatógeno. M. album, $M$. granulomatis y $M$. minus producen conidios ovoides a elipsoides como $M$. purpureogenum (Rombach et al. 1986, 1987; Sigler et al. 2010). Se distingue de $M$. album y M. minus por sus conidios más anchos (inferior a $3 \mu \mathrm{m}$ en las dos especies). Sin embargo, se distingue de $M$. granulomatis por las dimensiones de los conidios y la coloración de las masas conidiales de color gris verdoso en PDA (Nishi et al. 2017). Nuestra cepa de M. purpureogenum (similis), produjo un pigmento rojo-púrpura en medio de agar ya sea en cultivos duales y en cultivos puros en PDA, CYA, MEA y YES, una situación que no parece coincidir con lo observado por Nishi et al. (2017) que detectó esta coloración aparentemente solo en presencia dual. El pigmento podría tener algunas propiedades antifúngicas o ser un factor de virulencia contra insectos, al igual que la oosporeína, un pigmento rojo púrpura producido por Beauveria sp. (Feng et al. 2015). Debe resaltarse que nuestro aislamiento con altas similitudes con $M$. purpureogenum (no se conoce producción de pigmento púrpura en cultivo en otras especies del género) se ha diagnosticado solo por morfofisiología pudiendo ser una especie críptica, que al estudiarse por biología molecular muestre algunos cambios significativos genéticos que puedan alejarla o no de la especie diagnosticada (situación que se investigará en un trabajo posterior con biología molecular).

En el género Metarhizium, se han observado diferentes tipos de conidiogénesis. Todas las 
Dos interesantes contaminantes fúngicos - Piontelli E. et al .

formas son fialídicas y la morfología fialídica más predominante son sus formas cilíndricas de cuello corto como se especificó en Metarhizium en el siglo pasado. Más importante aún, la mayoría de las especies identificadas morfológicamente poseen estos caracteres que tienen una disposición similar a un candelabro de fiálides cilíndricas que forman un himenio compacto y pertenecen principalmente a los complejos de especies de $M$. anisopliae y $M$. flavoviride, patógenos de Coleoptera en el clado Coleoptera. En las ramas basales del clado Metarhizium, se encuentran fialides similares a los géneros Nomuraea y Paecilomyces. Todas las especies de Metarhizium forman conidióforos mononematosos excepto $M$. dendromatilis que es sinematoso (Rombach et al. 1987; Luangsa-ard y Alabama 2017).

Es un verdadero desafío distinguir especies de Metarhizium utilizando solo caracteres morfológicos, debido a que varias especies del género son especies morfológicamente crípticas (Bischoff et al.2009, Luangsa-ard y Alabama. 2017). El concepto definitorio de Metarhizium en las referencias mencionadas, radica solo en la producción de conidios que están predominantemente en varios tonos de verde, también pueden ser blancos o en tonos de marrón o amarillo, y en la producción de una empalizada de conidióforos con fiálides cilíndricas que forman una capa similar a himenio en un huésped artrópodo. La sistemática de Metarhizium, que es predominantemente asexual, tiene que depender en gran medida de enfoques multigénicos para estudiar la especiación críptica entre especies estrechamente relacionadas. Según lo descrito por Rombach et al. (1987), los principales criterios taxonómicos para el género Metarhizium son la forma de sus fiálides y conidios, con o sin zona de esporulación en una capa similar a himenio, así como un comportamiento de agrupamiento que forma masas aprismáticas de esporas. El tamaño de los conidios tiene gran importancia en la clasificación de Metarhizium, mientras el color de las hifas y de los conidios es de importancia secundaria.

Posiblemente, esta posible cepa chilena ha sufrido alguna variación genética por factores edáficos o adaptativos adquiridos en nuestros suelos, además no hay registros en la literatura nacional de su presencia lo que aumenta nuestro interés por conocer algunos factores asociados a su distribución geográfica.

Las infecciones en humanos por Metarhizium especialmente por $M$. anisopliae son raras, han surgido en los últimas dos décadas y probablemente son de tipo multifactorial, pero se han presentado en la literatura algunos casos en córnea, piel y uñas, sin embargo, el número de casos detectados nos lleva a pensar en el impacto de una nueva etapa en la taxonomía de Metarhizium en clínica, debido a que la sola morfología y algunos genes no ha permitido identificar las especies crípticas del género llevando a errores de clasificación de algunas de sus especies (Nourrisson et al. 217). El aislamiento de un dermatofito siempre confirma el patógeno, pero el problema surge para el personal del laboratorio cuando crece un hongo que no pertenece a este grupo y por ende debe distinguirse entre el contaminante y el verdadero patógeno. Se recomienda en estos casos seguir a Gupta et al. (2012) y Singal, A. et al, (2011). Si se aísla un género no integrante de los dermatofitos, se debe considerar como contaminante de laboratorio a menos que el $\mathrm{KOH}$ o la microscopía demuestre hifas atípicas en forma de fronda o si el mismo organismo es aislado repetidamente (Elewski. B.E. 1998).

Van Tieghem (1884), introdujo el género Monascus para 2 especies que producen ascomatas no ostiolados, $M$. ruber y $M$. mucoroides. La posición de Monascus (y las Monascaceae) a menudo se ubicó fuera del orden Eurotiales (Benny \& Kimbrough 1980, von Arx 1987, Stchigel \& Guarro 2007), pero los análisis filogenéticos ubican confidencialmente 
Dos interesantes contaminantes fúngicos - Piontelli E. et al.

este género en las Aspergillaceae (Eurotiales) (Berbee et al. 1995, Peterson 2008, Houbraken y Samson 2011, Vinnere-Pettersson et al. 2011). Se descubrió que el género Basipetospora es el anamorfo de Monascus y se caracteriza por la producción de aleurioconidios en forma basípeta de células conidiógenas indiferenciadas que se acortan progresivamente (retrogresión, Cole y Samson 1979). Los conidios de base trunca se asemejan a clamidosporas. Estas características distinguen a este género de los géneros Aspergillus y Penicillium relacionados filogenéticamente.

Actualmente, se conocen más de 30 especies, sin embargo, muchas de ellas son consideradas como sinónimos (Shao et al. 2014). La clasificación de Monascus se basa primariamente en características macro y microscópicas, como la pigmentación de las paredes cleistoteciales, los conidios y el crecimiento en medios de cultivos. Hawks-worth y Pitt (1983), revisaron el género basado en características fisiológicas y morfológicas y redujeron el número de especies aceptadas a tres: M. pilosus, $M$. ruber y $M$. purpureus. Desde ese estudio, diez nuevas especies fueron introducidas: $M$. albidulus, $M$. argentinensis, $M$. aurantiacus, $M$. eremophilus, $M$. floridanus, $M$. fumeus, M. lunisporas, $M$. pallens, M. rutilus y M. sanguineus (Cannon et al. 1995, Udagawa y Baba 1998, Stchigel y col. 2004, Li y Guo 2004). Con la descripción de estas especies, el género se convirtió morfológicamente más diverso, lo que sugiere gran diversidad genética. Por ejemplo, Monascus ruber crece rápidamente en medios de agar, M. lunisporas y $M$. pallens crecen de forma restringida y $M$. eremophilus es un xerófilo estricto y solo crece en medios con poca actividad de agua. Los esquemas de identificación basados en el fenotipo en Monascus fueron difíciles de igualar con los resultados obtenidos por ITS, la secuenciación parcial del gen LSU y / o $\beta$-tubulina (Park et al. 2004). Hoy en día, las especies se pueden delimitar por el genotipo, pero la aplicación de este concepto aún no se ha realizado y los resultados de tal análisis darán una idea de los límites de las especies. El género Monascus tiene importancia económica en varias áreas y varias especies se han utilizado ampliamente durante años en la producción de colorantes alimentarios amarillos y rojos y alimentos asiáticos fermentados, particularmente arroz rojo (angkak, angka, 'kójico rojo arroz'). El arroz rojo es de particular interés por su promoción de efectos en la salud (Shi \& Pan 2012) y, de hecho, la producción de compuestos con propiedades antibacterianas y estatinas reductoras del colesterol del tipo monacolina $\mathrm{K}$ (= mevinolina = lovastatina) se reportan en la especie $M$. pilosus, $M$. pubigerus, $M$. purpureus, $M$. ruber y M. vitreus (Vendruscolo y col. 2014). Sin embargo, especies de Monascus como M. anka, M. aurantiacus, M. kaoliang, M. pilosus, M. purpureus, $M$. ruber y $M$. sanguineus producen la micotoxina citrinina ( $\mathrm{Li}$ y col. 2015) y su presencia en los alimentos, incluido el arroz rojo, debe evitarse.

Las especies también rara vez se asocian con infecciones humanas. La mayoría de los hongos no dermatofitos, incluido $M$. ruber, están presentes en el medio ambiente y, por lo tanto, se debe tener cuidado al interpretar el cultivo del material infectado en especial en las dermatofitosis (Gupta et al. 2003, Dabet et al, 2016). M. ruber es una causa poco común de infección humana. Se sabe que causa infección gástrica asociada con el consumo de pescado seco y salado contaminado, ya que $M$. ruber tolera el cloruro de sodio (Lin YL et al, 2008; Samson et al. 2010). También se ha informado que causa alergia y anafilaxia en respuesta al arroz de levadura roja relacionado a una sensibilidad inmediata a Monascus purpureus (Rebeiro SM, et al, 1997). Otro estudio describió una infección renal directa por $M$. ruber después de la cirugía en un paciente de 70 años con múltiples cálculos renales (Sigler et al. 1999). 
Dos interesantes contaminantes fúngicos - Piontelli E. et al.

Los hongos del género Monascus son ascomicetos caracterizados por la producción de ascosporas resistentes al calor, que pueden sobrevivir a la pasteurización térmica. $M$. ruber se encuentra en el suelo y está relacionado con una contaminación de frutos post cosecha, y esta especie fue aislada por primera vez de aceitunas verdes pasteurizadas en Grecia (Panagou et al. 2002). En las aceitunas de mesa, el crecimiento de $M$. ruber se produce debido a su capacidad para adaptarse a determinadas condiciones, como crecer a tensiones de oxígeno muy bajas y sobrevivir a $\mathrm{pH}$ bajo y concentraciones de sal elevadas; En tales condiciones, $M$. ruber puede producir ascosporas resistentes al calor (Panagou et al. 2007). Estas condiciones permiten que el microorganismo se desarrolle en las aceitunas después de la cosecha, lo que puede ocurrir durante el almacenamiento y en las aceitunas de mesa enlatadas. Al conocer estos datos de la literatura revisamos la ficha clínica personal del paciente 2 y pudimos comunicarnos para averiguar si su trabajo guardaba cierta relación con las aceitunas y su producción y.... sorpresa, en su dieta estos frutos son su gran pasión.

\section{REFERENCIAS}

Alexander K.C. Leung, Joseph M. Lam, Kin F. Leong. et al. (2020). Onychomycosis: An Updated Review. Recent Patents on Inflammation \& Allergy Drug Discovery. Vol. 14, No. 133

Alexander Gómez-Sáenz y Roberto Arenas (2020). Onicomicosis mixta. Un caso por Trichophyton rubrum, Fusarium sp. y Candida albicans. Dermatología CMQ.18(1):48-50

Arx JA von (1987). A re-evaluation of the Eurotiales. Persoonia 13: 273-300.

Benny, GL and Kimbrough, JW. (1980). Synpsis of the order and families of Plectomycetes with key togenera . Mycotaxon 12:199
Barnard EL, Cannon PF (1987). A new species of Monascus from pine tissues in Florida. Mycologia 79: 479-484.

Berbee M.L. et a.l (1995). Is Penicillium monophyletic? An evaluation of phylogeny in the family Trichocomaceae from 18S, 5.8S and ITS ribosomal DNA sequence data. Mycologia, 87: 210222

Bischoff, J.F, Rehner, S, A, Humber, R.A. (2009) A multilocus phylogeny of the Metarhizium anisopliae lineage. Mycologia 101(4):512-530

Cannon PF, Abdullah SK, Abbas BA (1995). Two new species of Monascus from Iraq, with a key to known species of the genus. Mycological Research 99:659-662.

Chen, W., He, Y., Zhou, Y., Shao, Y., Feng, Y., Li, M., et al. (2015). Edible filamentous fungi from the species Monascus: early traditional fermentations, modern molecular biology, and future genomics. Compr. Rev.J. Food Sci. F. 14, 555-567

Cole GT, Samson RA. (1979). Patterns of development in conidial fungi. Pitman,London.

Dabet Rynga, Malini R Capoor, Swati Varshney and Ramesh V. (2016). Monascus ruber: A Rare Cause of Onychomycosis. J Infect Dis Pathol , 1:4

De A, Bose R, Kumar A, Mozumdar S. Targeted (2014). Delivery of Pesticides Using Biodegradable Polymeric Nanoparticles. Springer Briefs in Molecular Science; 2014.

Donald W.Roberts \& Raymond J.St. Leger. (2007) Metarhizium spp., Cosmopolitan Insect Pathogenic Fungi: Mycological Aspects. Advances in Applied MicrobiologyVolume 54, Pages 1-70

Driver, F. R R.J. Milner \& J.W.H. Trueman (2000). A taxonomic revision of Metarhizium based on a phylogenetic analysis of rDNA sequence data. Mycological Research, 104, pp. 134-150

Elewski, BE. (1998). Onychomycosis: Pathogenesis, diagnosis, and management. Clin Microbiol Rev 11:415-29 
Dos interesantes contaminantes fúngicos - Piontelli E. et al.

Feng Peng et al. (2015). Fungal biosynthesis of the bibenzoquinone oosporein to evade insect immunity. PNAS $8: 112$ (36) 11365-11370

Gupta AK, Ryder JE, Baran R, Summerbell RC (2003) Non-dermatophyte onychomy-chosis. Dermatol Clin 21: 257-268.

Gupta. AK, Drummond-Main C, Cooper EA, Brintnell W, Piraccini BM, Tosti A. (2012). Systematic review of nondermatophyte mold onychomycosis: Diagnosis, clinical types, epidemiology, and treatment. J Am Acad Dermatol. 66:494-502

Houbraken J, Samson RA (2011). Phylogeny of Penicillium and the segregation of Trichocomaceae into three families. Studies in Mycology 70: 1-51

Hawksworth DL, Pitt JI (1983). A new taxonomy for Monascus species based on cultural and microscopical characters. Australian Journal of Botany 31: 51-61.

He Y, Liu J, Chen Q, Gan S, Sun Tand Huo S. (2020) Monascus sanguineus May $\mathrm{Be}$ a Natural Nothospecies. Front. Microbiol. 11:614910

Kepler R.M. Humber, R.A. Bischoff J.F et al. (2014). Clarification of generic and species boundaries for Metarhizium and related fungi through multigene phylogenetics. Mycologia, 106,

Li Y-P, Tang XC, Wu W, et al. (2015). The ctnG gene encodes carbonic anhydrase involved in mycotoxin citrinin biosynthesis from Monascus aurantiacus. Food Additives \& Contaminants: Part A 32: $577-583$

Li ZQ, Guo F (2004). A further studies on the species of Monascus. Mycosystema 23: 1-6.6.

Lin YL, Wang TH, Lee MH, Su NW (2008) Biologically active components and neutraceuticals in the Monascus-fermented rice: a review. Appl Microbiol Biotechnol 77: 965-973.

Luangsa-ard, S. Mongkolsamrit, D. Thanakitpipattana et al. (2017). Clavicipitaceous entomopathogens: new species in Metarhizium and a new genus Nigelia.pp. 811-829
Mongkolsamrit. S, A. Khonsanit. D. Thanakitpipattan et al. (2020). Revisiting Metarhizium and the description of new species fromThailand. Sudies in Mycology 95: 171-251.

Nourrisson.C. D. Dupont.R.-A. Lavergne.J. Dorin.F. Forouzanfar.J. et al. (2017). Species of Metarhizium anisopliae complex implicated in human infections: retrospective sequencing study. Clinical Microbiology and Infection 23: pp.994-999.

López-Jodra Olga, Josep M Torres-Rodríguez Josep. (1999). Especies fúngicas poco comunes responsables de onicomicosis. Rev Iberoam Micol. 16: pp.11-15

Nishi Oumi, Susumu Shimizu \& Hiroki Sato (2017). Metarhizium bibionidarum and M. purpureo-genum: new species from Japan. Mycol Progress 16:987-998

Panagou, EZ, Katsaboxakis, CZ, Nychas G-JE. (2002). Heat resistance of Monascus ruber ascospores isolated from thermally processed green olives of the Conservolea variety. Int $\mathrm{J}$ Food Microbiol.76(1):11-18.

Panagou EZ, Kodogiannis V, Nychas GJE. (2007). Modelling fungal growth using radial basis function neural networks: the case of the ascomycetous fungus Monascus ruber van Tieghem. Int J Food Microbiol 117(3):276-286.

Park, H.G, E.K. Stamenova and S.C. Jong (2004). Phylogenetic relationships of Monascus species inferred from the ITS and the partial beta-tubulin gene. Botanical Bulletin of Academia Sinica, 45: 325-330

Peterson. SW (2008). Phylogenetic analysis of Aspergillus species using DNA sequences from four loci. Mycologia 100: 205-226.

Raymond R, Pihet EM. (2008). Conventional methods for the diagnosis of dermatophytosis. Mycopathologia. 166:295-306.

Rebeiro SM, Chagas GM, Campello AP, Kluppel ML (1997) Mechanism of citrinin-induced dysfunction of mitochondria. Effect on the homeostasis of the reactive oxygen species. Cell Biochem Funct 15: 203-209. 
Dos interesantes contaminantes fúngicos - Piontelli E. et al.

Rombach MC, Humber RA, Evans HC. (1986) Metarhizium album, a fungal pathogen of leaf-and planthoppers of rice. Transactions of the British Mycological Society 88, 451-459

Samson RA, Houbraken J, Thrane U, et al. (2010). Food and indoor fungi. In: CBS Laboratory manual series; No. 2. CBS-KNAW Fungal Biodiversity Centre, Utrecht, The Netherlands.

Shao, Y., Lei, M., Mao, Z., Zhou, Y., and Chen, F. (2014). Insights into Monascus biology at the genetic level. Appl. Microbiol. Biot. 98, 3911-3922

Shi Y-C, Pan T-M (2012). Red mold, diabetes, and oxidative stress: a review. Applied Microbiology and Biotechnology 94: 47-55

Sigler, L. et al. (2010). Disseminated mycoses in veiled Chameleons, caused by Chamaelomy-ces granulomatis a new fungus related tu Paecilomyces viridis. J. Cl. Microbiol. 48:3182-3192

Sigler, L., P. C. Kibsey, D. A. Sutton, S. P et al. (1999). Monascus ruber, causing renal infection, abstr. F-8, p. 297. Abstr. 99th Gen. Meet. Am. Soc. Microbiol. American Society for Microbiology, Washington, DC

Singal A, Khanna D. (2011). Onychomycosis: Diagnosis and management. Indian J Dermatol Venereol Leprol.77:659-72

Stchigel AM, Cano JF, Abdullah SK (2004). New and interesting species of Monascus from soil, with a key to the known species. Studies in Mycology50: 299-306

Thieghen P. van. (1884). Monascus genre noveau de lórdre des Ascomycetes. Bull. Soc. Bot. deFrance $31: 226-231$

Udagawa S, Baba H (1998). Monascus lunisporas, a new species isolated from mouldy feeds. Cryptogamie Mycologie 19: 269-276.

Vendruscolo F, Schmidell W, Ninow JL, et al. (2014). Antimicrobial activity of Monascus pigments produced in submerged fermentation. Journal of Food Processing and Preservation 38: 1860-1865.
Vinnere-Pettersson O, et al. (20011), Phylogeny and intraspecific variation of the extreme xerophile, Xeromyces bisporus.Fungal Biology, 115 :11001111

Xinggang Liao, Tammatha R. O'Brien, Weiguo Fang \& Raymond J. St. Leger. (2014) The plant beneficial effects of Metarhizium species correlate with their association with roots. Applied Microbiology and Biotechnology 98, pp.7089-7096 